\title{
TEORIA DEL GRAVIMETRO A ROTAZIONE
}

\author{
D. C. Borghi - E. Medi
}

1. Introduzione. - In una nota precedente è stato esposto un nuovo metodo per la misura assoluta della gravità: il rotogravimetro. Esso consiste nell'analizzare otticamente la superficie di rotazione generata rotando un recipiente, contenente un liquido, con un asse di rotazione verticale. Se il liquido è perfetto, la superficie è un paraboloide di rotazione, se invece esiste una tensione superficiale, si deve calcolare la nuova forma della superficie. Detto $(x, y)$ il piano di una sezione meridiana di detta superficie comprendente l'asse di rotazione $y$, la curva $y=y(x)$, sezione della superficie di rotazione anzidetta, yiace interamente sul piano $(x, y)$. In prima approssimazione, cioè non tenendo conto della tensione superficiale del liquido, questa curva è definita, com'è noto, da

$$
\frac{d y}{d x}=\frac{\stackrel{\leftrightarrow}{2}^{2}}{g} x
$$

Se ora si vuol tener conto anche della tensione superficiale del liquido, poiché la superficie del liquido rotante è una superficie di rotazione, basterà tener conto della "prima curvatura ) il cui raggio $P$ giace sul piano $(x, y)$, perché la seconda curvatura, introducendo il terzo asse, perpendicolare ad $(x, y)$, ̀̀ esclusa dal fatto che l'azimut non dà alcun contributo alla forma della sezione mediana della superficie di rotazione.

Perció, al posto della [0] metteremo

$$
\frac{d y}{d x}=2 G x+\frac{A \frac{d^{2} y}{d x^{2}}}{\sqrt{1+\left(\frac{d y}{d x}\right)^{2}}}
$$

con

$$
2 G=\frac{\omega^{2}}{g}
$$

Inoltre $A$ è una costante che dipende dalla natura del liquido; e 
il termine $\frac{d^{2} y}{d x^{2}} /\left[1+\left(\frac{d y}{d x}\right)^{2}\right]^{1 / 2}=\frac{1}{\rho}$ è linverso del raggio di curvatura della sezione meridiana in un punto di coordinate $x, y$.

Poniamo

$$
\left\{\begin{array}{l}
\frac{d y}{d x}=Z(x) \\
\frac{d^{*} y}{d x^{0}}=\frac{d Z(x)}{d x}
\end{array}\right.
$$

ed integriano [1] rispetto ad $x$ tra $x=0$ ed $x$

$$
y-y_{0}=G x^{2}+A \int_{0}^{x} \frac{\frac{d}{d x}}{\sqrt{1+L^{2}}} d x=G x^{*}+\int_{z}^{z_{0}} \frac{d Z}{\sqrt{1+z^{2}}}
$$

cioè

$$
y-y_{0}=G x+A(\operatorname{arcs} s h-C)
$$

$\operatorname{con}^{*}$

$$
C=\operatorname{arcSh} Z_{0}
$$

dove si sono posti con $y_{0}, Z_{0}$ i valori di $y$ e di $\frac{d y}{d z}$ per $x=0$.

La [t] mostra che la superficie di rotazione ha per sezione la curva di $1^{\circ}$ approssimazione [0], col termine correttivo $A$ (arc $S h Z-C$ ).

Da [1] e [3] risulta ovvio che per $Z$ vale lequazione differenziale

$$
Z-2 G x=4 \frac{\frac{d Z}{d x}}{\sqrt{1+Z^{2}}}
$$

Posto

$$
\iota=\frac{x}{-A} ; \quad B=2 G A ; \quad Z=\operatorname{Sh} \phi(t)
$$

la [6], indicando con apici le derivate rispetto a $t$, può scriversi

$$
S h \phi-B \prime=\emptyset^{\prime}(\prime)
$$

(*) Con Sh si indica il seno iperbolico. 
per cui la curva ricercata è definita da

$$
\left\{\begin{array}{l}
y-y_{0}+A C=G x^{2}+A \phi(t) \\
\emptyset^{\prime}(t)=S h \varnothing(t)-B t
\end{array}\right.
$$

s. Integrazione di [8].

Per integrare la [8], poniamo

$$
\phi(t)=\log f(t)
$$

cosi che [8] diviene, per definizione di $S h \phi$,

$$
2 \frac{f^{\prime}}{f}-t-\frac{1}{f}-2 B t
$$

ossia, moltiplicando per $4 f^{\prime \prime}$ ed ordinando,

$$
8 f f^{\prime}-4 f^{3}+4 f+8 B t f^{2}=0
$$

L'artificio di moltiplicare per $4 f^{-}$serve a far si che si possa separare il binomio $4 f^{3}-4 f$, il quale può rappresentarsi con una delle forme canoniche di funzioni ellittiche di Weierstrass, cioè, com’è noto,

$$
4 f^{3}-4 f=\mathrm{e}^{2}(+t-\because,-4 ; o) \quad(a=c t s t)
$$

Il doppio segno dell'argomento della p deriva dal fatto che questa è una funzione pari, e pertanto nulla cambia della definizione [11] se al posto di $t$ mettiano l'argomento

$$
\tau=|t|
$$

e scriveremo

$$
4 f^{3}-4 f=\rho^{\prime 2}\left(\tau-\tau_{0} ;-4 ; o\right) ; \quad \tau_{0}=\cos t .
$$

indicando con l'apice la derivata rispetto a $\tau$.

Ora, com"è noto, la posizione [13] è equivalente alla posizione*

$$
\begin{aligned}
& f=\rho\left(\tau-\tau_{0} ;-4 ; 0\right) \\
& \tau-\tau_{0}=\int_{\rho_{0}}^{\rho} \frac{d \rho}{\sqrt{\rho^{\prime 2}}}
\end{aligned}
$$

(") C.f. Whittaker-Witsox; Modern Analysis. 1950, n. 20, 221. 
Ciò vale a dire che $\tau-\tau_{0}$ è definita coi valori di p compatibili con l'equazione differenziale [10], perciò, sostituendo con [13] e [14] rispettivamente $f^{\prime}$ e le $f$ in [10] abbiamo (tralasciando per brevita gri argomenti)

$$
8 \varphi \sqrt{4 \varphi^{3}-4 \varphi^{2}}-\rho^{\prime 2}+8 B \tau \varphi^{2}=0
$$

ossia

$$
64 p^{2}\left(4 \varphi^{3}-4 \varphi^{0}\right)=\left(p^{2}-8 B \tau p^{2}=\left(4 p^{3}-4 \varphi^{2}-8 B \tau \varphi^{2}\right)^{2}\right.
$$

cioè, riducendo

$$
16\left(8^{3}-8\right)=\left(8^{2}-2 B \cdot \gamma^{2}-1\right)^{2}
$$

Svolgendo il quadrato ed ordinando, avremo la quartica a coefficienti antisimmetrici

$\varphi^{4}-(16+4 B \tau) \varphi^{3}-\left(2-4 B^{2} \tau^{2}\right) \rho^{2}+(16+4 B \tau) \varphi+1=0$

Dividendo [16] per $8^{\circ}$, essa può scriversi

$$
\left(\rho-\frac{1}{\varphi}\right)^{2}-\left(\rho-\frac{1}{\rho}\right)(16+4 B \tau)+4 B^{2} \tau^{2}=0
$$

cioè

$$
\rho-\frac{1}{\rho}=8+2 B \tau+\sqrt{4+2 B \tau}
$$

Se ora, nel nostro problema, si sceglie per origine degli assi il punto in cui la curva è tangente alla $x$, e da qui si alza l'asse della $y$, si vede che per $==0$ dev'essere $y=0$, quindi $\Phi=0$, quindi $f(o)=1$, e pertanto $\rho_{0}-\frac{1}{\rho_{0}}=0$. Perciò occorre scegliere in [17] la
radice

$$
\rho - \frac { 1 } { \varphi } = 8 + 2 B \tau - 4 \longdiv { 4 + 2 B \tau }
$$

che, appunto, si annulla per $\tau=0$. Posto

$$
\xi^{*}=4+2 B \tau
$$

la [18] si scriverà

$$
\vartheta-\frac{1}{8}=4-\dot{\zeta}^{4}-4 \xi^{2}=(2-\xi)^{2}=2 h
$$


con

$$
\sqrt{2} ;=2 \xi ; \xi=\sqrt{4+2 B \tau}
$$

Quindi $[18]$ diviene

$$
\rho^{2}-2 \circ h^{2}-1=0
$$

da cui

$$
p=h^{2} \sqrt{h^{4}-1-1} ; \quad n^{2}=\frac{1}{2}[2-\sqrt{4+2 B \tau}]^{2}
$$

Perché venga $\wp_{0}=1$, cosi da soddisfare la condizione $f_{0}=0$, bisogna in [23] scegliere la soluzione col segno + , quindi

$$
\wp=h^{2}+\sqrt{h^{1}+1}=\exp \operatorname{arcSh} h^{2}
$$

Dunque, essendo

$$
\tau-\tau_{0}=\int_{\varphi_{0}}^{\rho} \frac{d \varphi}{\sqrt{\rho^{\prime *}}}
$$

e dovendosi prendere per $p$ i valori compatibili con l'equazione differenziale [8], ossia i valori [24], ed essendo in particolare

$$
\rho_{0}=e^{\operatorname{arcSh} \frac{1}{2}\left(2-\xi_{0}\right)^{2}}=e^{\operatorname{arcSh} \frac{1}{2}(2-2)^{2}}=1
$$

si pui usare la trasformazione di $\left[14^{\prime}\right]$ in integrale ellittico di $1^{\text {a }}$ specie*

$$
i \overline{e_{1}-{ }_{3}}\left(\tau-\tau_{0}\right)=F(\theta ; \mu)
$$

dove $e_{1}>e_{2}>e_{3}$ sono le radiei alla Weierstrass di $4 \rho^{3}-4 \rho=0$ cioc̀

$$
e_{1}=+1 ; \quad e_{2}=0 ; \quad e_{3}=-1
$$

Liaromento $\theta$ è dato da

$$
\theta=\arcsin \sqrt{\frac{\omega_{1}-e_{3}}{\rho-e_{3}}}=\arcsin \sqrt{\frac{2}{\rho+1}}
$$

e il modulo $\mu$ da

$$
\mu=\sqrt{\frac{e_{1}-e_{2}}{e_{1}-e_{3}}}=\frac{1}{\sqrt{2}}
$$

(*) Cf. Sansoxe, Funz, di Variabile Complessa, II, 445. 
Perciò (")

$$
f=\gamma\left(\tau-\tau_{0} ;-4 ; 0\right)=e_{3}+\frac{e_{1}-e_{3}}{s n^{2}\left(\sqrt{e_{1}-e_{3}}\left(\tau-\tau_{0}\right) ; \mu\right)}
$$

dove $s n$ è il seno-amplitudine. Ponendo in [30] i valori [26]-[29] si ha:

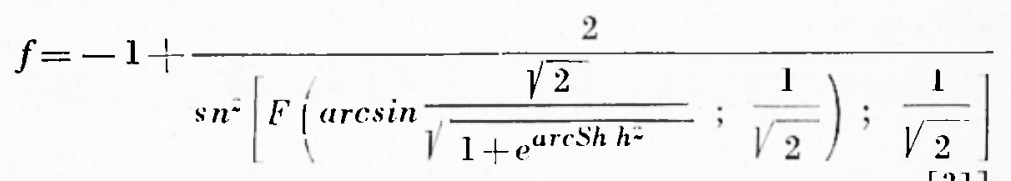

ricordando [9]

$$
f=-1+\frac{2}{\operatorname{sn}^{2}\left[F\left(\arcsin \frac{\sqrt{2}}{\sqrt{1-h^{2}+l^{2} h^{4}+1}} ; \frac{1}{\sqrt{2}}\right) ; \frac{1}{\sqrt{2}}\right]}
$$

Per [21] si ha $h=0$ per $\xi=2$, ciò, per [19], per $\tau=0$.

Per tale valore si ha

$$
f_{0}=-1+\frac{2}{; n^{2}\left[F\left(\frac{\pi}{2} ; \frac{1}{\sqrt{2}}\right) ; \frac{1}{\sqrt{2}}\right]}=-1+2=+1=\phi_{0}
$$

come dovevasi aspettare. Per ogni altro $\tau>0$, la funzione $s n^{2}:$ minore di 1 , quindi si ha

$$
f>1
$$

\section{3. - Determinazione di G.}

Abbiamo dunque trovato che, con $y_{0}=0$, la sezione della superficie di rotazione generata rotando il liquido (v. $\$$ l) è data dalla curva

$$
y=G x^{2}+A \lg \left[2 n s^{2}\left(\theta ; \frac{1}{\sqrt{2}}\right)-1\right]
$$

(*) C.fr. il formulario per le funzioni ellittiche in Jahnke Emo, Funktionentajeln, ed. Dover, 1945, p. 100. Puo stabilirsi un collegamento tra la funzione $f$ e le funzioni lemniscate; ef. Whitraker e Warson, op. cit., p. 52. L'na tabulazione della funzione sn è quella a 5 decimali di L. M. Mreve.Tronson, Jacobian Elliptic Functions Tables, ed. Dover 1950. Per l'integrale ellittico $F(0 ; \mu)$ si veda la tabulazione della classica opera di JaHNke-Embf. Funktionentafeln, p. 62 ss., che dà pure la bibliografia per tabulazioni più precise (p. 83). 


$$
\begin{gathered}
\operatorname{con} n s(0)=\frac{1}{\operatorname{sn}(\theta)} \mathrm{e} \operatorname{con} \\
\theta=F\left(\arcsin \frac{\sqrt{2}}{\sqrt{1+h^{*}+\sqrt{h^{4}+1}}} ; \frac{1}{\sqrt{2}}\right) \\
h^{2}=\frac{1}{2}(2-\sqrt{4-26|x|})^{2}
\end{gathered}
$$

perche $B \tau=G|x|$

Immaginiamo ora di eseguire due misure, di due punti $\left[y_{1} x_{1}\right]$, $\left[y_{2} x_{2}\right]$ della curva [34]. Si può allora eliminare senzaltro il parametro $A$ perche si ha (tralasciando il modulo $\frac{1}{\sqrt{2}}$ )

$$
\frac{y_{1}-G x_{1}^{2}}{y_{2}-G x_{2}^{2}}-\frac{\lg \left(2 n s^{2} \theta_{1}-1\right)}{\lg \left(2 n s^{2} \theta_{2}-1\right)}=L(G)
$$

dove $0_{1}$ e $0_{2}$ sono $i$ valori ricavati da $[35]$ in cui si mettono rispettivamente $i$ valori $\mid x_{1}$ ed $\left|x_{2}\right|$.

La [37] risolve formalmente il problema della misura di $G$, essendo un'equazione (trascendente) con la sola incognita $G$.

Ora si osservi che se per le ascisse $x_{1}$ ed $x_{0}$ si prendono due valori noti e fissi (che siano anche convenienti dal punto di vista sperimentale), allora il secondo membro di [37] diviene una funzione della sola $G$, cioè

$$
\frac{y_{1}-G x_{1}^{2}}{y_{1}-G x_{2}^{2}}=L(G)
$$

Percio, eseguita la misura dei due punti $\left[x_{1} y_{1}\right]\left[x_{2} y_{2}\right]$, e supposta tabulata la funzione $L(G)$ per $i$ due valori-base $x_{1}$ ed $x_{. \prime}$, la misura di $G$ si ha per quel valore di $G$ che soddisfa la [38].

Roma - Istituto Nazionale di Geofisica --. Ottobre 1954

\section{RI.ASSUNTO}

In un precedente lavoro ì stato esposto un nuovo metodo per la determinazione del valore assoluto della gravità esso consiste nel determinare la distanza focale di un paraboloide di rivoluzione. ottenuto facendo ruotare un liquido intorno ad un asse verticale, e misurando la velocita angolare del sistema ruotante. 
La tensione superficiale del liquido è una causa di errore nella misura. Introducendo lespressione di tale perturbazione nella equazio. ne del paraboloide. si ha una equazione differenziale, che viene risolta nel presente lavoro. La soluzione esatta permette anche dal punto di vista sperimentale la completa soluzione del problema generale.

\section{$S T M M R Y$}

In a preceding work, "new method was discussed for the determination of the absolute valne of the constant of gravity: the method consists in measuring the focal distance of a parabaloid of revolution. obtained by rotating a liquid around a vertical axis, and measuring the angular velocity of the rotating system.

The surface tension of the liquid is a cause of error in the measurement. Introducing the expression for this perturbation into the equation for the parabaloid gives a differential equation "hich is solved in the present work. The exact solution allows, from the experimental point of vien, a complete solution of the general problem. 